\title{
Editorial
}

\section{Rehabilitación y manejo del dolor}

La rehabilitación es el cuidado que se debe recibir para recuperar, mantener o mejorar las capacidades que se necesitan para la vida diarial.

En relación al dolor crónico, este genera más discapacidad que la suma de las discapacidades de las enfermedades cardiovasculares, cerebrovasculares, cáncer y SIDA.

Esta problemática se traduce en restricciones en la participación social y laboral, limitaciones en actividades de la vida diaria e instrumentales, alteraciones cognitivas, emocionales y conductuales, con el consiguiente deterioro en la calidad de vida de las personas que lo padecen ${ }^{2}$.

En este escenario la rehabilitación y el manejo del dolor son inseparables, siendo el manejo del dolor un pilar fundamental para llevar a cabo el proceso rehabilitador, cuyo objetivo será reducir al mínimo las consecuencias funcionales, psíquicas, sociales y económicas (licencias médicas, pérdida de ganancia permanente entre otros) de las deficiencias o discapacidades producidas por el dolor y que los pacientes alcancen el máximo nivel de independencia funcional, integración en la sociedad y calidad de vida ${ }^{3}$.

Se ha avanzado considerablemente en el conocimiento de la fisiopatología del dolor y contamos con más y mejores herramientas diagnósticas y terapéuticas, pero aún quedan grandes interrogantes y desafios.

El seguir investigando para determinar la prevalencia del dolor en patologías discapacitantes, profundizar el conocimiento de los distintos sindromes dolorosos nos da las bases para incluir en nuestra conducta terapéutica la pesquisa y manejo adecuado de la patología dolorosa.

La comprensión de los procesos involucrados en el dolor crónico nos obliga a realizar planes de manejo donde el enfoque rehabilitador es fundamental. Se debe potenciar la prevención, el autocuidado, las terapias psicológicas y el ejercicio como pilares principales del tratamiento.

El manejo farmacológico e intervencional debe ser usado en forma racional y como herramienta para lograr estos objetivos. Se debe mantener una reevaluación continua con base a los avances funcionales y las mejoras en la calidad de vida de nuestros pacientes. Siendo el dolor un tema de gran prevalencia en las patologías discapacitantes, debemos liderar su estudio e investigación constante, promover el trabajo en equipo, la educación como base de los planes de tratamiento, pesquisa precoz de patología dolorosa crónica, una visión preventiva en pacientes con factores de riesgo y su derivación oportuna a unidades de manejo especializado cuando se requiera.

En este número esperamos contribuir a avanzar en este tremendo y hermoso desafío como rehabilitadores.

Dra. Ana Luisa Miranda Monsalve Jefa Unidad de Tratamiento del Dolor HCUCH. Fisiatra Unidad de Dolor Clínica Alemana de Santiago. Profesor Asistente Universidad de Chile y Universidad del Desarrollo. 


\section{Referencias bibliográficas}

1. Andersson L, Rozo A. Rol del médico rehabilitador en el manejo del dolor. Rev Col Med Fis Rehab 2015; 25 63-9.

2. Stanos SP, McLean J, Rader L. Physical medicine rehabilitation: Approach to pain. Med Clin N Am 2007; 91: 57-95.
3. Fine PG. Long-Term consequences of chronic pain: mounting evidence for pain as a neurological disease and parallels with other chronic disease States. Pain Medicine 2011; 12: 996-1004.

4. Rice D, McNair P, Huysmans E, Letzen J, Finan P. Best evidence rehabilitation for chronic pain Part 5: Osteoarthritis. J Clin Med 2019; 8,1769. doi:10.3390/ jem8111769. 